\title{
Single transverse spin asymmetry of forward neutrons
}

\author{
B. Z. Kopeliovich, I. K. Potashnikova, and Iván Schmidt \\ Departamento de Física, Universidad Técnica Federico Santa María; and \\ Instituto de estudios avanzados en ciencias en ingeniera; and \\ Centro Científico-Tecnológico de Valparaíso; \\ Casilla 110-V, Valparaíso, Chile \\ J. Soffer \\ Department of Physics, Temple University, Philadelphia, PA 19122-6082, USA
}

\begin{abstract}
We calculate the single transverse spin asymmetry $A_{N}(t)$, for inclusive neutron production in $p p$ collisions at forward rapidities relative to the polarized proton in the energy range of RHIC. Absorptive corrections to the pion pole generate a relative phase between the spin-flip and non-flip amplitudes, leading to a transverse spin asymmetry which is found to be far too small to explain the magnitude of $A_{N}$ observed in the PHENIX experiment. A larger contribution, which does not vanish at high energies, comes from the interference of pion and $a_{1}$-Reggeon exchanges. The unnatural parity of $a_{1}$ guarantees a substantial phase shift, although the magnitude is strongly suppressed by the smallness of diffractive $\pi p \rightarrow a_{1} p$ cross section. We replace the Regge $a_{1}$ pole by the Regge cut corresponding to the $\pi \rho$ exchange in the $1^{+} S$ state. The production of such a state, which we treat as an effective pole $a$, forms a narrow peak in the $3 \pi$ invariant mass distribution in diffractive $\pi p$ interactions. The cross section is large, so one can assume that this state saturates the spectral function of the axial current and we can determine its coupling to nucleons via the PCAC Goldberger-Treiman relation and the second Weinberg sum rule. The numerical results of the parameter-free calculation of $A_{N}$ are in excellent agreement with the PHENIX data.
\end{abstract}

PACS numbers: 13.85.Ni, 11.80.Cr, 11.80.Gw, 13.88.+e

\section{INTRODUCTION}

The single transverse spin asymmetry of neutrons was measured recently by the PHENIX experiment at RHIC [1] in $p p$ collisions at energies $\sqrt{s}=62,200$ and $500 \mathrm{GeV}$. The measurements were performed with a transversely polarized proton beam and the neutron was detected at very forward and backward rapidities relative to the polarized beam. Preliminary results are depicted in Fig. 1. An appreciable single transverse spin asymmetry was found in events with large fractional neutron momenta $z$. The data agree with a linear dependence on the neutron transverse momentum $q_{T}$, and different energy match well, what indicates at an energy independent $A_{N}\left(q_{T}\right)$.

Usually polarization data are more sensitive to the mechanisms of reactions than the cross section. Below we demonstrate that the large magnitude of the single transverse spin asymmetry of forward neutrons discovered in [1], reveals a new important mechanism of neutron production ignored in all previous studies of the reaction cross section.

At the same time, neutrons produced with $x_{F}<0$ show a small asymmetry, consistent with zero. This fact is explained by the so called Abarbanel-Gross theorem [2] which predicts zero transverse-spin asymmetry for particles produced in the fragmentation region of an unpolarized beam. This statement was proven within the Regge pole model illustrated in Fig. 2. The amplitude of the reaction $p \uparrow+p \rightarrow X+n$ squared, Fig. 2a, is related by the optical theorem with the triple-Regge graph in Fig. $2 \mathrm{~b}$. According to Regge factorization the proton spin can cor-

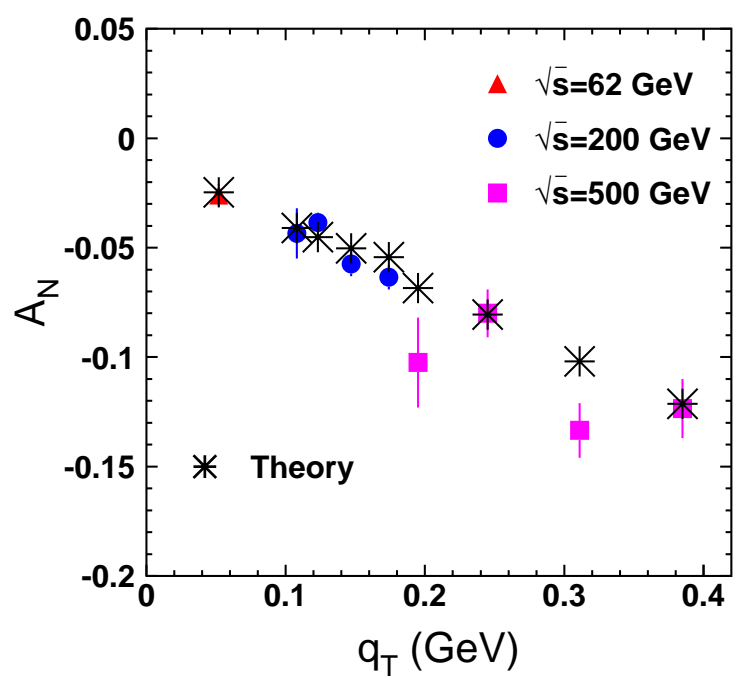

FIG. 1: (Color online) Single transverse spin asymmetry $A_{N}$ in the reaction $p p \rightarrow n X$, measured at $\sqrt{s}=62,200,500 \mathrm{GeV}$ [1] (preliminary data). The asterisks show the result of our calculation, Eq. (38), which was done point by point, since each experimental point has a specific value of $z$ (see Table \).

relates only with the vector product, $\left[\vec{k} \times \vec{k}^{\prime}\right]$, of the proton momenta in the two conjugated amplitudes, as is shown in Fig. 2b. According to the optical theorem these momenta are equal, $\vec{k}=\vec{k}^{\prime}$, so no transverse spin correlation is possible. Regge cuts shown in Fig. 2k breakdown this statement, but the magnitude of the gained single-spin asymmetry calculated in [3], turned out to be very small, less than $1 \%$. 


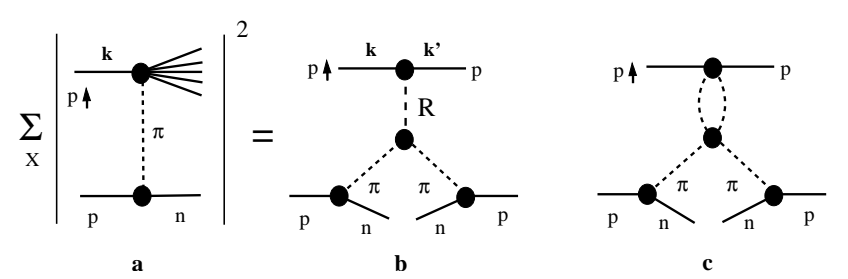

FIG. 2: Graphical representation of the cross section of inclusive reaction $p \uparrow+p \rightarrow X+n$ with a polarized proton beam and neutron produced in the fragmentation region of the unpolarized proton, and the related triple Regge graph (a-b). The Regge cut correction to the previous graph (c).

\section{SPIN STRUCTURE OF THE PION POLE}

The importance of the pion pole for neutron production has been known since the pioneering paper [4]. Its Reggeized version for leading neutrons in DIS was proposed in [5]. A further development, which includes the spin structure of the amplitudes was published in 7]. Contrary to the conventional wisdom that the pionnucleon vertex is pure spin-flip, a large non-flip term in the amplitude appears if $z<1$. In Born approximation the pion exchange in neutron production, depicted in Fig. 3, in the leading order in the small parameter $m_{N} / \sqrt{s}$ has the form [7],

$$
A_{p \rightarrow n}^{B}(\vec{q}, z)=\bar{\xi}_{n}\left[\sigma_{3} q_{L}+\frac{1}{\sqrt{z}} \vec{\sigma} \cdot \vec{q}_{T}\right] \xi_{p} \phi^{B}\left(q_{T}, z\right)
$$

where $\vec{\sigma}$ are the Pauli matrices; $\xi_{p, n}$ are the proton or neutron spinors; $\vec{q}_{T}$ and

$$
q_{L}=\frac{1-z}{\sqrt{z}} m_{N}
$$

are the transverse and longitudinal components of the momentum transfer respectively.

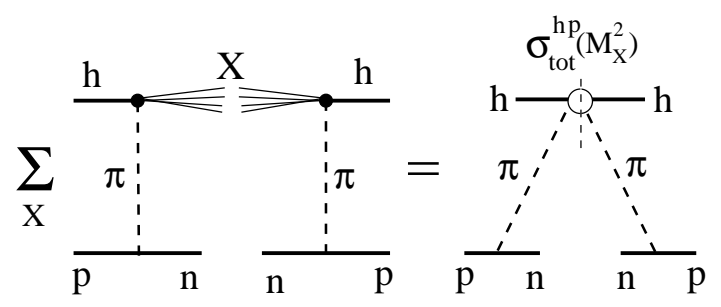

FIG. 3: Graphical representation of the cross section of inclusive neutron production in hadron-proton collisions, in the fragmentation region of the proton.

At large $z$ the pseudoscalar amplitude $\phi^{B}\left(q_{T}, z\right)$ has the Regge form [5],

$$
\begin{aligned}
\phi^{B}\left(q_{T}, z\right) & =\frac{\alpha_{\pi}^{\prime}}{8} G_{\pi^{+} p n}(t) \eta_{\pi}(t)(1-z)^{-\alpha_{\pi}(t)} \\
& \times A_{\pi^{+} p \rightarrow X}\left(M_{X}^{2}\right)
\end{aligned}
$$

where $M_{X}^{2}=(1-z) s$; the 4-momentum transfer squared $(t)$ has the form,

$$
-t=q_{L}^{2}+\frac{1}{z} q_{T}^{2}
$$

and $\eta_{\pi}(t)$ is the phase (signature) factor,

$$
\eta_{\pi}(t)=i-\operatorname{ctg}\left[\frac{\pi \alpha_{\pi}(t)}{2}\right]
$$

The second term contains the pion pole,

$$
\operatorname{Re} \eta_{\pi}(t) \approx \frac{2}{\pi \alpha_{\pi}^{\prime}} \frac{1}{m_{\pi}^{2}-t}
$$

In what follows we assume that the pion Regge trajectory is a linear function of $t, \alpha_{\pi}(t)=\alpha_{\pi}^{\prime}\left(t-m_{\pi}^{2}\right)$, where $\alpha_{\pi}^{\prime} \approx$ $0.9 \mathrm{GeV}^{-2}$.

The effective vertex function is parametrized as,

$$
G_{\pi^{+} p n}(t)=g_{\pi^{+} p n} e^{R_{\pi}^{2} t}
$$

where the pion-nucleon coupling $g_{\pi^{+} p n}^{2} / 8 \pi=13.85$. The $t$-slope parameter $R_{\pi}^{2}$ incorporates the $t$-dependences of the coupling and of the $\pi N$ inelastic amplitude. Although it is not well known, its value is not really important for us, since we concentrate on the small $t$ region. For further calculations we fix $R_{\pi}^{2}=4 \mathrm{GeV}^{-2}$, which is naturally related to the nucleon size.

The amplitude (11) is normalized as $M_{X}^{2} \sigma_{t o t}^{\pi^{+} p}=$ $\sum_{X}\left|A_{\pi^{+} p \rightarrow X}\left(M_{X}^{2}\right)\right|^{2}$. Correspondingly, the differential cross section of inclusive neutron production reads,

$$
\begin{aligned}
z \frac{d \sigma_{p \rightarrow n}^{B}}{d z d q_{T}^{2}} & =\left(\frac{\alpha_{\pi}^{\prime}}{8}\right)^{2}|t| G_{\pi+p n}^{2}(t)\left|\eta_{\pi}(t)\right|^{2} \\
& \times(1-z)^{1-2 \alpha_{\pi}(t)} \sigma_{\text {tot }}^{\pi^{+} p}\left(M_{X}^{2}\right) .
\end{aligned}
$$

This pure pion pole model has two obvious shortcomings: (i) the cross section Eq. (8) substantially overshoots data [7]; (ii) no single transverse spin asymmetry is possible because the spin-flip and non-flip terms in the amplitude (11) have no phase shift.

\section{SPIN EFFECTS GENERATED BY INITIAL/FINAL STATE INTERACTIONS}

It is known that initial and final state interactions (ISI/FSI) can generate spin effects [6], if they are absent in the Born approximation. In the process under discussion ISI/FSI essentially modify the spin amplitudes of the process, suppressing the magnitude of the cross section and generating a nonzero relative phase shift.

Absorptive corrections look simpler and factorize in impact parameter. Therefore, we perform a Fourier transform on the amplitude (1) to impact parameter representation, multiply it by the survival probability amplitude $S(b, z)$,

$$
f_{p \rightarrow n}(b, z)=f_{p \rightarrow n}^{B}(b, z) S(b, z),
$$


and then return back to the momentum representation

where

[7]. The Born amplitude (1) in impact parameter reads,

$$
f_{p \rightarrow n}^{B}(\vec{b}, z)=\bar{\xi}_{n}\left[\sigma_{3} q_{L} \theta_{0}^{B}(b, z)-i \frac{\vec{\sigma} \cdot \vec{b}}{\sqrt{z} b} \theta_{s}^{B}(b, z)\right] \xi_{p},
$$

$$
\begin{aligned}
& \theta_{0}^{B}(b, z)=\int d^{2} q e^{i \vec{b} \vec{q}} \phi^{B}\left(q_{T}, z\right)=N_{X}^{\pi}(z)\left\{i \frac{\pi \alpha_{\pi}^{\prime}}{2 z \beta^{2}} K_{0}(b / \beta)+\frac{1}{1-\beta^{2} \epsilon^{2}}\left[K_{0}(\epsilon b)-K_{0}(b / \beta)\right]\right\} \\
& \theta_{s}^{B}(b, z)=\frac{1}{b} \int d^{2} q e^{i \vec{b} \vec{q}}(\vec{b} \cdot \vec{q}) \phi^{B}\left(q_{T}, z\right)=N_{X}^{\pi}(z)\left\{i \frac{\pi \alpha_{\pi}^{\prime}}{2 z \beta^{3}} K_{1}(b / \beta)+\frac{1}{1-\beta^{2} \epsilon^{2}}\left[\epsilon K_{1}(\epsilon b)-\frac{1}{\beta} K_{1}(b / \beta)\right]\right\} ;
\end{aligned}
$$

and

$$
\begin{array}{rl}
N_{X}^{\pi}(z)=\frac{1}{2} g_{\pi+p n} & z(1-z)^{\alpha_{\pi}^{\prime}\left(m_{\pi}^{2}+q_{L}^{2}\right)} e^{-R_{\pi}^{2} q_{L}^{2}} \\
\epsilon^{2}=z\left(q_{L}^{2}+m_{\pi}^{2}\right), \\
\beta^{2}=\frac{1}{z}\left[R_{\pi}^{2}-\alpha_{\pi}^{\prime} \ln (1-z)\right] .
\end{array}
$$

As is illustrated in Fig. 3, in the rapidity interval covered by the pion, $\Delta y=|\ln (1-z)|$, no particles are produced. Since inelastic ISI/FSI of the spectator partons tend to fill this rapidity gap, its survival probability leads to a suppression. It was found in [7] that the partonic system, which participates in ISI/FSI with the target nucleon, is a 5-quark color octet-octet dipole. The amplitude suppression factor $S(b, z)$ was found in [7] to be rather small, leading to a strong reduction of the cross section of leading neutron production. The magnitude of the partial cross section, which is expected to be universal, was found to agree well with ZEUS data for neutron produced in DIS [8], and with NA49 data for $p p$ collisions [9]. Although these data confirm the shape of the $z$ distribution measured earlier at ISR [10], the latter overestimated the normalization.

Following (9) we applied the absorption factor to the Born spin amplitudes Eq. (10), and performing Fourier transfer back to momentum representation, arrive at,

$$
A_{p \rightarrow n}(\vec{q}, z)=\bar{\xi}_{n}\left[\sigma_{3} q_{L} \phi_{0}\left(q_{T}, z\right)-i \vec{\sigma} \cdot \vec{q}_{T} \frac{\phi_{s}\left(q_{T}, z\right)}{\sqrt{z}}\right] \xi_{p},
$$

where

$$
\begin{aligned}
\operatorname{Re} \phi_{0}\left(q_{T}, z\right) & =\frac{N_{X}^{\pi}(z)}{2 \pi\left(1-\beta^{2} \epsilon^{2}\right)} \int_{0}^{\infty} d b b J_{0}\left(b q_{T}\right) \\
& \times\left[K_{0}(\epsilon b)-K_{0}(b / \beta)\right] S(b, z) ; \\
\operatorname{Im} \phi_{0}\left(q_{T}, z\right) & =\frac{\alpha_{\pi}^{\prime} N_{X}^{\pi}(z)}{4 z \beta^{2}} \int_{0}^{\infty} d b b J_{0}\left(b q_{T}\right)
\end{aligned}
$$

$$
\begin{aligned}
& \times K_{0}(b / \beta) S(b, z) \\
\operatorname{Re} \phi_{s}\left(q_{T}, z\right) & =\frac{N_{X}^{\pi}(z)}{2 \pi\left(1-\beta^{2} \epsilon^{2}\right) q_{T}} \int_{0}^{\infty} d b b J_{1}\left(b q_{T}\right) \\
& \times\left[\epsilon K_{1}(\epsilon b)-\frac{1}{\beta} K_{1}(b / \beta)\right] S(b, z) ; \\
\operatorname{Im} \phi_{s}\left(q_{T}, z\right) & =\frac{\alpha_{\pi}^{\prime} N_{X}^{\pi}(z)}{4 z \beta^{3} q_{T}} \int_{0}^{\infty} d b b J_{1}\left(b q_{T}\right) \\
& \times K_{1}(b / \beta) S(b, z) .
\end{aligned}
$$

We are now in a position to calculate the single transverse spin asymmetry,

$$
\begin{aligned}
A_{N}\left(q_{T}, z\right) & =\frac{2 q_{T} q_{L} \sqrt{z} \sum_{X}\left|\phi_{0}\left(q_{T}, z\right)\right|\left|\phi_{s}\left(q_{T}, z\right)\right|}{z q_{L}^{2} \sum_{X}\left|\phi_{0}\left(q_{T}, z\right)\right|^{2}+q_{T}^{2} \sum_{X}\left|\phi_{s}\left(q_{T}, z\right)\right|^{2}} \\
& \times \sin \left(\delta_{s}-\delta_{0}\right),
\end{aligned}
$$

where

$$
\tan \delta_{0, s}=\frac{\operatorname{Im} \phi_{0, s}\left(q_{T}, z\right)}{\operatorname{Re} \phi_{0, s}\left(q_{T}, z\right)} .
$$

The results are depicted in Fig. 4, It is clear that our calculations significantly underestimate the PHENIX data in the range of $q_{T}$ covered by the experiment. 


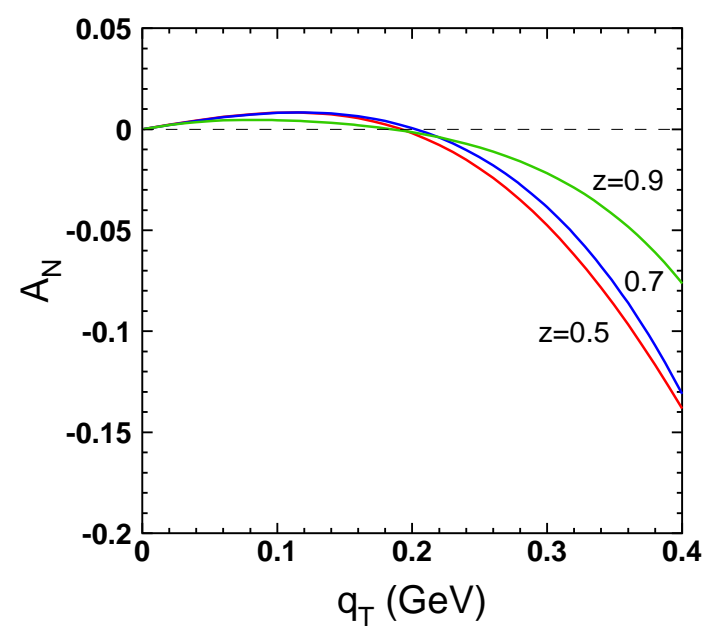

FIG. 4: (Color online) Single transverse spin asymmetry of leading neutrons related to single pion exchange corrected for absorptive corrections, as function of $q_{T}$. The curves from bottom to top correspond to $z=0.5,0.7$ and 0.9 .

\section{OTHER SOURCES OF SPIN EFFECTS: $a_{1}$ REGGEON?}

In addition to pion exchange, other Regge poles $R=$ $\rho, a_{2}, \omega, a_{1}$, etc. and Regge cuts can contribute to the $p p \rightarrow n X$ reaction. We should remind that the total collision energy is shared by the reaction $\pi+p \rightarrow R+p$ depicted in the upper blob of the diagram Fig. (5), and by the rapidity gap, rather unequally: while the former can be very large, $M_{X}^{2}=(1-z) s$ at high energies, the c.m. energy for pion exchange is rather small, $s^{\prime}=s_{0} /(1-z)$, and independent of $s$.

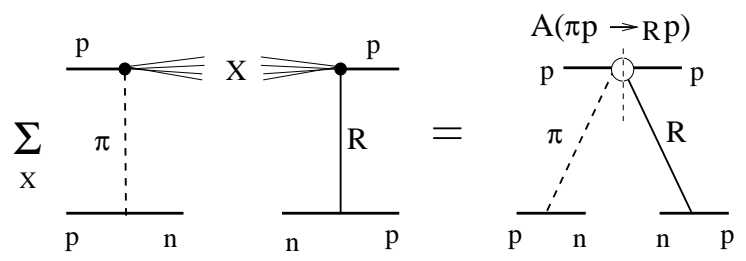

FIG. 5: Graphical representation for the interference between the amplitudes with pion and Reggeon exchanges.

Summing over different produced states $X$ and using completeness one arrives at the imaginary part of the amplitude of the process $\pi+p \rightarrow R+p$ at c.m. energy $M_{X}^{2}$. The production of natural parity states, like $\rho, a_{2}$, etc. can proceed only via reggeon exchange, therefore these amplitudes are strongly suppressed at RHIC energies by a power of $M_{X}$ (dependent on the Regge intercept) and can be safely neglected everywhere, except at the region of very small $(1-z) \sim s_{0} / s$, unreachable experimentally.

Only the unnatural parity states, which can be diffractively produced by a pion, like the $a_{1}$ meson, or $\rho$ - $\pi$ in the axial vector or pseudo-scalar states, contribute to the interference term in the neutron production cross section at high energies.

The $a_{1} N N$ vertex is known to be pure non spin-flip [11, 12]. Therefore, it should be added to the first term in Eq. (15),

$$
A_{p \rightarrow n}^{a_{1}}\left(q_{T}, z\right)=e_{\mu}^{L} \bar{n} \gamma_{5} \gamma_{\mu} p=\frac{2 m_{N} q_{L}}{\sqrt{|t|}} \phi_{0}^{a}\left(q_{T}, z\right) \bar{\xi}_{n} \sigma_{3} \xi_{p},
$$

where the longitudinal polarization vector of $a_{1}$ reads [13],

$$
e_{\mu}^{L}=\frac{1}{\sqrt{|t|}}\left(\sqrt{q_{0}^{2}-t}, 0,0, q_{0}\right)
$$

and the transferred energy

$$
q_{0}=E_{p}-E_{n}=q_{L}+O\left(m_{N} / \sqrt{s}\right) .
$$

In the Born approximation,

$$
\begin{aligned}
\phi_{0}^{a}\left(q_{T}, z\right) & =\frac{\alpha_{a_{1}}^{\prime}}{8} G_{a^{+} p n}(t) \eta_{a_{1}}(t) \\
& \times(1-z)^{-\alpha_{a_{1}}(t)} A_{a_{1}^{+} p \rightarrow X}\left(M_{X}^{2}\right),
\end{aligned}
$$

and

$$
\eta_{a_{1}}(t)=-i-\operatorname{tg}\left[\frac{\pi \alpha_{a_{1}}(t)}{2}\right] .
$$

The amplitude (22) contains three unknowns, to be fixed before numerical evaluation:

- The amplitude $A_{a_{1}^{+} p \rightarrow X}\left(M_{X}^{2}\right)$;

- The $a_{1}$-nucleon vertex $G_{a_{1}^{+} p n}(t)$;

- The Regge trajectory $\alpha_{a_{1}}(t)$.

Notice that the general structure of the amplitude Eq. (25) is valid for any axial-vector state. In what follows we find the $a_{1}$ pole to be quite a weak singularity, and conclude that the spectral function of the axial current is dominated by the contribution of $\pi \rho$ pair in the $1^{+} S$ state. So we will replace the weak $a_{1}$ pole by an effective singularity $a$.

\section{A. Diffractive production of $a_{1}$ meson and non-resonant $\pi \rho\left(1^{+} S\right)$ pairs}

The amplitude $A_{a_{1}^{+} p \rightarrow X}\left(M_{X}^{2}\right)$ is normalized as,

$$
\begin{aligned}
& \sum_{X} A_{a_{1}^{+} p \rightarrow X}^{\dagger}\left(M_{X}^{2}\right) A_{\pi p \rightarrow X}\left(M_{X}^{2}\right)=4 \sqrt{\pi} M_{X}^{2} \\
\times & \sqrt{d \sigma\left(\pi p \rightarrow a_{1} p\right) /\left.d p_{T}^{2}\right|_{p_{T}=0}}
\end{aligned}
$$

Evaluation of the ratio of the forward diffractive-toelastic cross sections in this expression is a subtle problem. The cross section of diffractive $a_{1}$ production is so 
small that it has been escaping detection for a long time. Eventually $a_{1}$ production was observed in $\pi+p \rightarrow 3 \pi+p$ performing a phase shift analysis of high-statistics data $[14,15]$. The $a_{1}$ resonance was only detected in this process as a fast variation of the relative phase of $1^{+} S$.

The $\rho \pi\left(1^{+} S\right)$ mass distribution depicted in Fig. 6 forms a strong and narrow peak, related mainly to the Deck mechanism [16]. The data [15] combined for lab

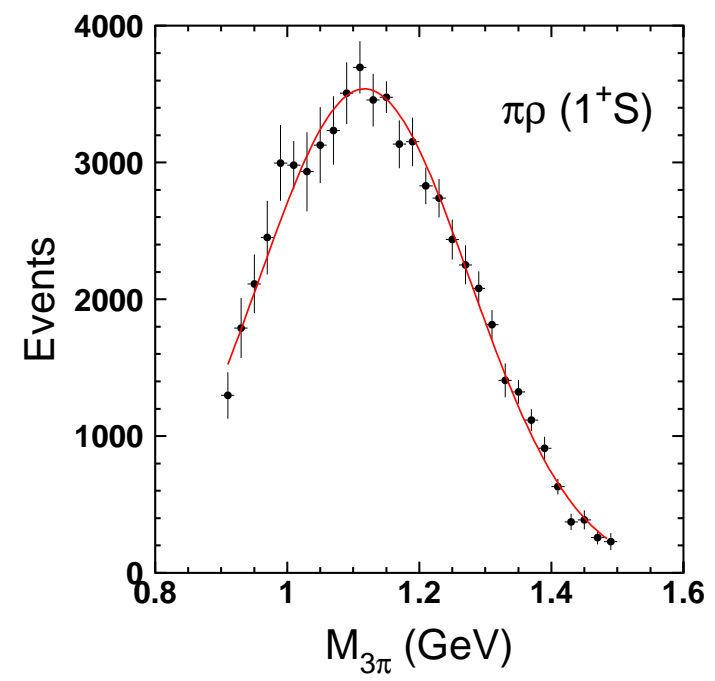

FIG. 6: (Color online) Variation of $1^{+} S$ wave intensity with $3 \pi$ invariant mass, for $q_{T}^{2}<0.05 \mathrm{GeV}^{2}$. Data for $E_{l a b}=$ 63 and $94 \mathrm{GeV}$ are summed [15]. The curve is fitted with parametrization (28).

energies 63 and $94 \mathrm{GeV}$ were taken with the small- $q_{T}$ trigger. We normalize this distribution on the measured cross section in the invariant mass interval $1.0<$ $M_{3 \pi}<1.2 \mathrm{GeV}$ which is $54 \pm 8 \mu b$ and $48 \pm 7 \mu b$ at 63 and $94 \mathrm{GeV}$ respectively. With the measured $p_{T}^{2}$-slope $B=14.3 \pm 0.17 \mathrm{GeV}^{-2}$ we can evaluate the forward cross section for this mass interval at $d \sigma /\left.d p_{T}^{2}\right|_{p_{T}=0}=$ $0.73 \pm 0.07 \mathrm{mb} / \mathrm{GeV}^{2}$ averaged for the two energies.

In order to normalize the mass distribution plotted in Fig. 6 to this cross section, we fitted the data with a simple Gaussian parametrization,

$$
\left.\frac{d \sigma}{d M_{3 \pi} d q_{T}^{2}}\right|_{q_{T}=0}=\lambda \exp \left[-\frac{\left(M_{3 \pi}-m_{a}\right)^{2}}{\Gamma_{a}^{2}}\right]
$$

with parameters $m_{a}=(1.117 \pm 0.004) \mathrm{GeV}, \Gamma_{a}=(0.226 \pm$ $0.004) \mathrm{GeV}$, and $\lambda=3.91 \pm 0.03 \mathrm{mb} / \mathrm{GeV}^{3}$. We also made a $10 \%$ correction for the nonzero values of $q_{T}^{2}<$ $0.05 \mathrm{GeV}^{2}$ of data in Fig. 6. Notice that the central mass of the peak is significantly smaller than $m_{a_{1}}=1.28 \mathrm{GeV}$ [17.

Eventually, integrating the normalized distribution over the invariant $3 \pi$ mass under the $a$-peak in Fig. 6 we arrive at the $\pi \rho\left(1^{+} S\right)$ forward production cross section,

$$
\left.\frac{d \sigma(\pi p \rightarrow \pi \rho p)}{d p_{T}^{2}}\right|_{1+S, p_{T}=0}=1.67 \mathrm{mb} / \mathrm{GeV}^{2}
$$

Now we have to extrapolate the diffractive cross section Eq. (29), from the energy range of the fixed target experiments [15], $s_{1} \sim 119-177 \mathrm{GeV}^{2}$ up to the substantially higher c.m. energies $M_{X}^{2}=(1-z) s$ of RHIC. We extrapolate the single diffractive cross section with the following energy dependence,

$$
\frac{d \sigma_{s d}\left(M_{X}^{2}\right)}{d q_{T}^{2}}=\frac{d \sigma_{s d}\left(s_{1}\right)}{d q_{T}^{2}}\left(\frac{M_{X}^{2}}{s_{1}}\right)^{0.16} \frac{K_{\pi p}\left(M_{X}^{2}\right)}{K_{\pi p}\left(s_{1}\right)}
$$

where $K(s)$ is the survival probability of a large rapidity gap, which in the eikonal approximation has the form [18]

$$
\begin{aligned}
K_{\pi p}(s) & =1-\frac{1}{\pi} \frac{\sigma_{t o t}^{\pi p}(s)}{B_{s d}^{\pi p}(s)+2 B_{e l}^{\pi p}(s)} \\
& +\frac{1}{(4 \pi)^{2}} \frac{\left[\sigma_{t o t}^{\pi p}(s)\right]^{2}}{B_{e l}^{\pi p}(s)\left[B_{s d}^{\pi p}(s)+B_{e l}^{\pi p}(s)\right]} .
\end{aligned}
$$

For the energy dependent total $\pi p$ cross section $\sigma_{\text {tot }}^{\pi p}(s)$ we rely on the parametrization given in [17]. For the elastic and single diffractive slopes we use $B^{\pi p}(s)=$ $B_{0}+2 \alpha_{\mathbb{P}}^{\prime} \ln \left(s / s_{0}\right)$, where $B_{0}=6 \mathrm{GeV}^{-2}$ for elastic and $B_{0}=9 \mathrm{GeV}^{-2}$ for single diffractive processes. $\alpha_{\mathbb{P}}^{\prime}=$ $0.25 \mathrm{GeV}^{-2}$.

Notice that the replacement of the $a_{1}$ pole in the dispersion relation by the effective singularity $a$ also helps to settle several problems related to the assumed $a_{1}$ dominance for the axial current. In particular, this assumption leads to so called Piketty-Stodolsky paradox [13, 19]. Namely, based on the PCAC hypothesis, which relates the pion pole and heavier states contributions in the dispersion relation for the axial current, one arrives at the conclusion that diffractive $\pi \rightarrow a_{1}$ and elastic $\pi \rightarrow \pi$ cross sections should be equal, while experimentally they are different by more than one order of magnitude. Replacing the $a_{1}$ pole by the effective singularity $a$ solves the problem [13, 20 22].

Summarizing, we found that the production cross section for the $a_{1}$ meson is too small to produce a sizable contribution to neutron production. It should be replace by the more significant production of a $\pi \rho 1^{+} S$ state, which forms a narrow resonance-like peak in the $3 \pi$ invariant mass distribution. Thus, we introduce an effective "pole" $a$ in the dispersion relation for the axial-vector current, and predict its production cross section in $\pi p$ collisions at high energies.

\section{B. $a N N$ coupling}

Like for the pion we parametrize the $a_{1}$-nucleon vertex in Eq. (25) as $G_{a^{+} p n}(t)=g_{a^{+} p n} \exp \left(R_{a}^{2} t\right)$. The slope parameter $R_{a}^{2}$ is poorly known, however in the small $t$ region under consideration it is not of great importance. Like for the pion vertex, it is natural to expect the slope to be related to the nucleon size, so we fix $R_{a}^{2}=R_{\pi}^{2}=$ $4 \mathrm{GeV}^{2}$ for further calculations. 
The $a$-nucleon coupling $g_{a^{+} p n}$ can be estimated based on PCAC. Although it is tempting to interpret the Goldberger-Treiman relation and Adler theorem as pion pole dominance, the pion pole does not contribute in either $\beta$-decay or high-energy neutrino interactions, because of conservation of the lepton current (neglecting the lepton mass) [13, 19, 23]. In order to have PCAC heavy axial states contributing to the dispersion relation for the amplitude of the process must miraculously reproduce the pion pole. If we replace the combined contribution of the heavy state by an affective pole $a[13,20,21]$, the Goldberger-Treiman relation relates the $a$ and pion poles,

$$
\frac{\sqrt{2} f_{a} g_{a N N}}{m_{a}^{2}}=\frac{f_{\pi} g_{\pi N N}}{\sqrt{2} m_{N}}
$$

In the second Weinberg sum rule the spectral function of the vector current can be represented by the $\rho$ meson pole. Correspondingly, the axial spectral function is saturated by the $a$ meson, because the pion does not contribute to the second Weinberg sum rule. Then one arrives at the relation,

$$
f_{a}=f_{\rho}=\frac{\sqrt{2} m_{\rho}^{2}}{\gamma_{\rho}}
$$

where $\gamma_{\rho}$ is the universal coupling $(\rho N N, \rho \pi \pi$, etc), $\gamma_{\rho}^{2} / 4 \pi=2.4$.

Thus, for the $a$ to pion couplings ratio we get,

$$
\frac{g_{a N N}}{g_{\pi N N}}=\frac{m_{a}^{2} f_{\pi}}{2 m_{N} f_{\rho}} \approx 0.5 \text {. }
$$

A different expression was derived in [12], based on the first Weinberg sum rule, which includes the pion pole,

$$
\frac{g_{a N N}}{g_{\pi N N}}=\frac{m_{a} m_{\rho} f_{\pi}}{2 m_{N}\left(f_{\rho}^{2}-m_{\rho}^{2} f_{\pi}^{2}\right)^{1 / 2}} .
$$

Numerically the results (34) and (35) are very close in the chiral limit of massless pion. Otherwise they differ by $15 \%$.

\section{Regge trajectory of the "a-pole"}

So far the narrow $a$-peak in the spectral function of the axial current could be treated as an effective pole replacing the real one $a_{1}$, which was found to be too weak. However, the Regge singularity in the complex angular momentum plane, related to the $\pi$ - $\rho$ exchange, is a Regge cut rather than a pole. The trajectory of the cut can be expressed in terms of the $\pi$ and $\rho$ Reggeons,

$$
\alpha_{\pi \rho}(t)=\alpha_{\pi}(0)+\alpha_{\rho}(0)-1+\frac{\alpha_{\pi}^{\prime} \alpha_{\rho}^{\prime}}{\alpha_{\pi}^{\prime}+\alpha_{\rho}^{\prime}} t .
$$

For further numerical evaluations we fix $\alpha(0)=1 / 2$ and $\alpha_{\pi}^{\prime}=\alpha_{\rho}^{\prime}=0.9 \mathrm{GeV}^{-2}$, so $\alpha_{\pi \rho}(t)=-0.5+0.45 t$.
Correspondingly, the phase (signature) factor for the unnatural parity $a$-Reggeon exchange reads,

$$
\eta_{a}(t)=-i-\operatorname{tg}\left[\pi \alpha_{a}(t) / 2\right],
$$

where $\alpha_{a}(t)=\alpha_{\pi \rho}(t)$.

This factor provides a significant phase shift $\Delta \phi=\pi / 4$ relative to the pion, and this phase shifts rises with $t$ up to the maximal value of $\pi / 2$ at $t=-1 \mathrm{GeV}^{2}$. This interference looks like a promising source of a significant single transverse spin asymmetry.

Notice that the intercept of the $\pi$ - $\rho$ cut turns out to be rather close to the intercept of the $a_{1}$ Regge pole, $\alpha_{a_{1}}(0)=-0.43$, which corresponds to a straight Regge trajectory with the universal slope crossing the position of the $a_{1}$ pole on the Chew-Frautschi plot.

\section{SINGLE TRANSVERSE SPIN ASYMMETRY GENERATED BY $\pi$ - $a$ INTERFERENCE}

Eventually, we are in a position to perform a parameter free calculation of the $a-\pi$ interference contribution to single transverse spin asymmetry of neutron production,

$$
\begin{gathered}
A_{N}\left(q_{T}, z\right)=q_{T} \frac{4 m_{N} q_{L}}{|t|^{3 / 2}}(1-z)^{\Delta \alpha(t)} \frac{\operatorname{Im} \eta_{\pi}^{*}(t) \eta_{a}(t)}{\left|\eta_{\pi}(t)\right|^{2}} \\
\times \frac{g_{a^{+} p n}}{g_{\pi^{+} p n}}\left(\frac{d \sigma_{\pi p \rightarrow a p}\left(M_{X}^{2}\right) /\left.d p_{T}^{2}\right|_{p_{T}=0}}{d \sigma_{\pi p \rightarrow \pi p}\left(M_{X}^{2}\right) /\left.d p_{T}^{2}\right|_{p_{T}=0}}\right)^{1 / 2},
\end{gathered}
$$

where $\Delta \alpha(t)=\alpha_{\pi}(t)-\alpha_{a}(t)$.

The specific value of $z$ is important for the factors that appear in (38), in particular for $t$ given by (2)-(44). We extracted values of $z$ for each experimental point shown in Fig. (1) from data [1] as is described in Appendix A and presented in Table I. The results of the calculation with Eq. (38) are plotted in Fig. 1 by asterisks. The agreement with data is excellent, but we estimate the uncertainty of the normalization of the theoretical results at about $30 \%$.

Notice that in these calculations we neglected the absorptive corrections, because they have very little influence on $A_{N}$, Eq. (38). Indeed, as we have seen above, they cause a tiny variation of the relative phase, which can be neglected. It was also demonstrated in [7] that the spin-flip and non-flip amplitudes are suppressed by absorption similarly, because the kinematics of the data is rather far from the pion pole (see Table \). We checked that inclusion of absorption leaves the asymmetry well within the theoretical uncertainty of the calculations.

Although the PHENIX experiment also provided data for $A_{N}$ with a special selection of charged hadrons detected at pseudo-rapidities $3.0<\eta<3.9$, we do not think that comparison with these data would bring new valuable information. Indeed, in this case summing over the intermediates states $X$ one cannot rely on completeness any more, and the theoretical uncertainties would increase significantly. 


\section{SUMMARY}

The results of the the PHENIX experiment at RHIC on single transverse spin asymmetry of neutrons produced in polarized $p p$ collisions at very forward rapidities have brought precious information about the mechanisms of leading neutron production. The naturally expected pion pole calculated in the Born approximation does not explain either the magnitude of the cross section, nor the observed azimuthal asymmetry. The absorptive corrections, which can be interpreted as the survival probability of a rapidity gap, substantially reduce the cross section improving agreement with data. At the same time, the relative phase shift between the spin-flip and non-flip amplitudes generated by absorption turns out to be far too small to explain the measured single transverse spin asymmetry.

Another possible source of a considerable spin effect considered here is the interference between the amplitude of neutron production via pion and $a_{1}$ Reggeon exchanges. Because $a_{1}$ has unnatural parity, it can be produced diffractively in $\pi+p \rightarrow a_{1}+p$, so is not suppressed at high c.m. energy $M_{X}$. It also provides a large, close to maximal, relative phase shift between the non-flip $a_{1}$ and spin-flip pion exchange amplitudes.

It turns out, however, that the $a_{1}$ exchange contribution is strongly suppressed by the smallness of the diffractive $a_{1}$ resonance production. Nevertheless, we found that it is possible to replace this resonance by $\pi \rho$ in the unnatural parity $1^{+} S$ state, because it forms a narrow peak in the $3 \pi$ invariant mass distribution, so can be treated as an effective pole, named $a$, in a dispersion relation for the axial current.

This allows to determine the $a$-nucleon coupling using PCAC, which relates the contributions of heavy states (saturated by the $a$ pole and the pion pole). Additional information about the leptonic decay constant of $a$ is obtained from the second Weinberg sum rule.

Although the $\pi \rho$ exchange corresponds to a Regge cut, rather than a pole, we found its Regge intercept to be rather close to the one for $a_{1}$ Reggeon, so the phase shift is similar as well.

Finally, we calculated the single transverse spin asymmetry at different values of the kinematic variables, $s, q_{T}$ and $z$, and found very good agreement with data.

\section{ACKNOWLEDGMENTS}

We are thankful to Elke Aschenauer and Yousef Makdisi for providing information about the new PHENIX results on neutron single transverse spin asymmetry. This work was supported in part by Fondecyt (Chile) grants 1090236, 1090291 and 1100287, and by ConicytDFG grant No. 084-2009.

\section{Appendix A. FRACTIONAL NEUTRON MOMENTA IN PHENIX DATA}

We extracted values of $z$ for each experimental point shown in Fig. 1] from data [1], using the relation with the scattering angle, $q_{T}=z \sin \theta \sqrt{s} / 2$. The results for all points are presented in Table I]

TABLE I: Values of angles of neutron detection, of neutron transverse momenta, and deduced values of fractional neutron momenta $z$, in the results of the PHENIX measurements [1] of the neutron single transverse spin asymmetry.

\begin{tabular}{|c|c|ccccc|cccc|}
\hline$\sqrt{s}(\mathrm{GeV})$ & 62 & \multicolumn{5}{|c|}{200} & \multicolumn{5}{|c|}{500} \\
\hline$\theta(\mathrm{mrad})$ & 1.89 & 1.38 & 1.57 & 1.87 & 2.21 & 1.083 & 1.36 & 1.74 & 2.15 \\
\hline$q_{T}(\mathrm{GeV})$ & 0.05 & 0.11 & 0.12 & 0.15 & 0.18 & 0.20 & 0.25 & 0.31 & 0.39 \\
\hline$z$ & 0.89 & 0.78 & 0.78 & 0.79 & 0.79 & 0.72 & 0.73 & 0.72 & 0.72 \\
\hline
\end{tabular}

[1] K. Tanida [PHENIX Collaboration], J. Phys. Conf. Ser. 295 (2011) 012097.

[2] H. D. I. Abarbanel and D. J. Gross, Phys. Rev. Lett. 26, 732 (1971); Phys. Rev. D 5, 699 (1972).

[3] B. Z. Kopeliovich, L. I. Lapidus and A. B. Zamolodchikov, Sov. J. Nucl. Phys. 26, 209 (1977) [Yad. Fiz. 26, 399 (1977)].

[4] M. Bishari, Phys. Lett. B 38, 510 (1972).

[5] B. Kopeliovich, B. Povh and I. Potashnikova, Z. Phys. C 73, 125 (1996).

[6] S. J. Brodsky, D. S. Hwang, I. Schmidt, Phys. Lett. B530, 99-107 (2002). hep-ph/0201296.

[7] B. Z. Kopeliovich, I. K. Potashnikova, I. Schmidt and J. Soffer, Phys. Rev. D 78, 014031 (2008) arXiv:0805.4534 [hep-ph]].

[8] S. Chekanov et al. [ZEUS Collaboration], Nucl. Phys. B 637, 3 (2002).
[9] D. Varga, NA49 Collaboration, Eur. Phys. J. C 33, S515 (2004).

[10] W. Flauger and F. Mönnig, Nucl. Phys. B109 (1976) 347.

[11] J. D. Kimel and J. F. Owens, Nucl. Phys. B 122, 464 (1977).

[12] H. E. Haber and G. L. Kane, Nucl. Phys. B 129, 429 (1977).

[13] B. Z. Kopeliovich and P. Marage, Int. J. Mod. Phys. A 8, 1513 (1993).

[14] Yu. M. Antipov et al., Nucl. Phys. B 63, 153 (1973) [Erratum-ibid. B 76, 574 (1974)].

[15] C. Daum et al. [ACCMOR Collaboration], Nucl. Phys. B 182, 269 (1981).

[16] R. T. Deck, Phys. Rev. Lett. 13, 169 (1964).

[17] K. Nakamura et al. (Particle Data Group), J. Phys. G 37, 075021 (2010).

[18] B. Z. Kopeliovich, I. K. Potashnikova, I. Schmidt 
and A. V. Tarasov, Phys. Rev. D 74, 114024 (2006) arXiv:hep-ph/0605157.

[19] C. A. Piketty and L. Stodolsky, Nucl. Phys. B 15, 571 (1970).

[20] A. A. Belkov and B. Z. Kopeliovich, Sov. J. Nucl. Phys. 46, 499 (1987) [Yad. Fiz. 46, 874 (1987)].

[21] B. Z. Kopeliovich, I. K. Potashnikova, I. Schmidt and M. Siddikov, Phys. Rev. C 84, 024608 (2011)
arXiv:1105.1711 [hep-ph]].

[22] B. Z. Kopeliovich, I. Schmidt and M. Siddikov, Phys. Rev. D 84, 033012 (2011) arXiv:1107.2845 [hep-ph]].

[23] J. S. Bell, In Cumming, J. (ed.): Osborn, H. (ed.): Hadron interactions of electrons and photons, Academic Press, 369-394 (1971). 